\title{
Computing the minimal solutions of finite fuzzy relation equations on linear carriers
}

\author{
Juan Carlos Díaz-Moreno, Jesús Medina, Esko Turunen \\ Department of Mathematics \\ University of Cádiz, Spain \\ Email: \{juancarlos.diaz,jesus.medina\}@uca.es \\ Research Unit Computational Logic. \\ Vienna University of Technology. Wien, Austria \\ Email: esko.turunen@tut.fi
}

\begin{abstract}
Fuzzy relation equations is an important tool for managing and modeling uncertain or imprecise datasets, which has useful applied to, e.g. approximate reasoning, time series forecast, decision making, fuzzy control, etc. This paper considers a general fuzzy relation equation, which has minimal solutions, if it is solvable. In this case, an algebraic characterization is introduced which provides an interesting method to compute minimal solutions in this general setting.
\end{abstract}

\section{INTRODUCTION}

$\mathbf{F}$ UZZY relation equations were introduced by E. Sanchez in the seventies [11]. These equations have widely been studied in different papers [1], [3], [6]. For example, they have proven that the set of solutions of solvable fuzzy relation equations is a upper-preserving complete lattice in which the greatest solutions is completely determined. Nevertheless, the computation of minimal solutions is not so direct. These solutions have also been studied in several papers [2], [4], [10], [12], [17], [15], [16], [18], [13] and several algorithms have been developed, but in restrictive frameworks, restrictions that limit the flexibility of the possible applications.

Hence, first of all, it is fundamental to study general frameworks in which the minimal solutions of each solvable fuzzy relation equation exist and that each solution will be between the greatest solution and a minimal solution.

This paper considers a general setting, in which the operators may neither be commutative nor associative and they only need to be monotone and residuated inf-preserving mappings of non-empty sets on the right argument. The linearity of the carrier, together with the inf-preserving property, ensures the existence of minimal solutions whenever a solution exists.

Mainly, this paper introduces a procedure in order to obtain the minimal solutions of a solvable of the introduced general fuzzy relation equations. Moreover, we have presented a detailed algorithm to compute these important solutions, together with several illustrative examples.

\section{GENERAL FUZZY RELATION EQUATIONS}

Throughout this paper we will consider a complete linear lattice $(L, \preceq)$, in which the bottom and the top elements exist and they are denoted as 0,1 , respectively. Given a set $V$, the ordering $\preceq$ in the lattice induces a partial order on the set of
$L$-fuzzy subsets of $V, L^{V}$. This ordering provides to $L^{V}$ the structure of a complete lattice.

A general residuated operator will also be used in this paper to define the fuzzy relation equation, as in [8]. This residuated operator will be denoted as $\odot: L \times L \rightarrow L$, which is order preserving in both arguments and there exists another operator $\rightarrow: L \times L \rightarrow L$, satisfying the following adjoint property with the conjunctor $\odot$

$$
x \odot y \preceq z \quad \text { if and only if } y \preceq x \rightarrow z
$$

for each $x, y, z \in L$. This property is equivalent to say that $\odot$ preserves supremums in the second argument; $x \odot \bigvee\{y \mid y \in$ $Y\}=\bigvee\{x \odot y \mid y \in Y\}$, for all $Y \subseteq L$.

These operators, as were noted in [8], generalize other kind of residuated pairs [7], [5], since only the monotonicity and the adjoint property are considered.

Definition 1. Given the pair $(\odot, \rightarrow)$, a fuzzy relation equation is the equation:

$$
R \circ X=T,
$$

where $R: U \times V \rightarrow L, T: U \times W \rightarrow L$ are given finite $L$ fuzzy relations and $X: V \times W \rightarrow L$ is unknown; and $R \circ$ $X: U \times W \rightarrow L$ is defined, for each $u \in U, w \in W$, as

$$
(R \circ X)\langle u, w\rangle=\bigvee\{R\langle u, v\rangle \odot X\langle v, w\rangle \mid v \in V\} .
$$

It is well known that the fuzzy relation equation (2) has a solution if and only if

$$
(R \Rightarrow T)\langle v, w\rangle=\bigwedge\{R\langle u, v\rangle \rightarrow T\langle u, w\rangle \mid u \in U\}
$$

is a solution and, in that case, it is the greatest solution, see [7], [11], [14].

\section{COMPUTING MiNimAL SOLUTIONS ON LINEAR LATTICES}

Definition 2. Given an operator $\odot: L \times L \rightarrow L$, we will say that it holds the IPNE-condition (making reference to that $\odot$ is Infimum Preserving of arbitrary Non-Empty sets), if it verify

$$
a \odot \bigwedge B=\bigwedge\{a \odot b \mid b \in B\}
$$

for each element $a \in L$ and each non-empty subset $B \subseteq L$. 
From now on, let us consider a general solvable fuzzy relation equation (2), where $R, X, T$ are finite and $\odot$ satisfies the IPNE-condition.

First of all, the auxiliary sets $V_{u w}$ need to be introduced, which are associated with the elements $u \in U, w \in W$ and the greatest solution $R \Rightarrow T$. Since for each $u \in U, w \in W$

$$
\bigvee\{R\langle u, v\rangle \odot(R \Rightarrow T)\langle v, w\rangle \mid v \in V\}=T\langle u, w\rangle,
$$

$L$ is linear and $V$ is finite, there exists at least one $v_{s} \in V$ validating the equation

$$
R\left\langle u, v_{s}\right\rangle \odot(R \Rightarrow T)\left\langle v_{s}, w\right\rangle=T\langle u, w\rangle .
$$

Therefore, the set

$$
V_{u w}=\{v \in V \mid R\langle u, v\rangle \odot(R \Rightarrow T)\langle v, w\rangle=T\langle u, w\rangle\}
$$

is not empty and, for all $v \notin V_{u w}$, the strict inequality $R\langle u, v\rangle \odot(R \Rightarrow T)\langle v, w\rangle\langle T\langle u, w\rangle$ holds.

Each $v_{s}$ in $V_{u w}$ will provide a fuzzy subset $S_{u w s}$ as follows: Given $v_{s} \in V_{u w}$, we have that

$$
\left\{d \in L \mid R\left\langle u, v_{s}\right\rangle \odot d=T\langle u, w\rangle\right\} \neq \varnothing
$$

and the infimum $\bigwedge\left\{d \in L \mid R\left\langle u, v_{s}\right\rangle \odot d=T\langle u, w\rangle\right\}=e_{s}$ also satisfies the equality

$$
R\left\langle u, v_{s}\right\rangle \odot e_{s}=T\langle u, w\rangle
$$

by the IPNE-condition. These elements are used to define the fuzzy subsets of $V, Z_{u w s}: V \rightarrow L$, defined by

$$
Z_{u w s}(v)=\left\{\begin{array}{cc}
e_{s} & \text { if } v=v_{s} \\
0 & \text { otherwise }
\end{array}\right.
$$

which form the set $Z_{u w}$, that is $Z_{u w}=\left\{Z_{u w s} \mid v_{s} \in V_{u w}\right\}$, for each $u \in U, w \in W$. These sets will be used to characterize the set of solutions of Equation (2) by the notion of covering.

Theorem 3. The L-fuzzy relation $X: V \times W \rightarrow L$ is a solution of a solvable Equation (2) if and only if $X \preceq(R \Rightarrow T)$ and, for each $w \in W$, the fuzzy subset $X_{w}: V \rightarrow L$, defined by $X_{w}(v)=X\langle v, w\rangle$, is a cover of $\left\{Z_{u w} \mid u \in U\right\}$.

As a consequence, the minimal solutions are characterized by the minimal covers.

Corollary 4. $X: V \times W \rightarrow L$ is a minimal solution of Equation (2) if and only if, for each $w \in W, X_{w}: V \rightarrow L$, defined by $X_{w}(v)=X\langle v, w\rangle$, is a minimal cover of $\left\{Z_{u w} \mid u \in U\right\}$.

Hence, from the corollary above, minimal solutions of the fuzzy relation equation (2) are obtained from $R \Rightarrow T$. Next, the detailed algorithms are introduced.

Module MINIMAL_COVERING uses an usual algorithm in order to compute minimal covering of subsets.

Example III.1. Let us assume the standard MV-algebra [9], that is, $L=[0,1]$ is the unit interval, $\odot: L \times L \rightarrow L$ is the Eukasiewicz operator defined by $x \odot y=\max \{0, x+y-1\}$ and $\rightarrow: L \times L \rightarrow L$ its residuated implication, defined by $y \rightarrow z=\min \{1,1-y+z\}$, for all $x, y, z \in[0,1]$. input : Universes $U, V$ and $W$, the fuzzy relations $R: U \times V \rightarrow L$ and $T: U \times W \rightarrow L$

output: $M S S=$ Set of minimal solutions of the fuzzy relation equation $R \circ X=T$

$1 M S S:=[]$;

$2 S:=R \Rightarrow T$, which is the greatest solution of $R \circ X=T$;

\begin{tabular}{|c|c|c|c|c|c|c|c|c|}
\hline$R$ & $v_{1}$ & $v_{2}$ & $v_{3}$ & \multirow{3}{*}{ and } & $T$ & $w_{1}$ & $w_{2}$ & $w_{3}$ \\
\hline$u_{1}$ & 0.9 & 0.5 & 0.9 & & $u_{1}$ & 0.8 & 0.4 & 0.7 \\
\hline$u_{2}$ & 0.2 & 0.9 & 0.7 & & $u_{2}$ & 0.6 & 0.7 & 0.3 \\
\hline$u_{3}$ & 0.8 & 0.6 & 0.9 & & $u_{3}$ & 0.8 & 0.4 & 0.6 \\
\hline
\end{tabular}

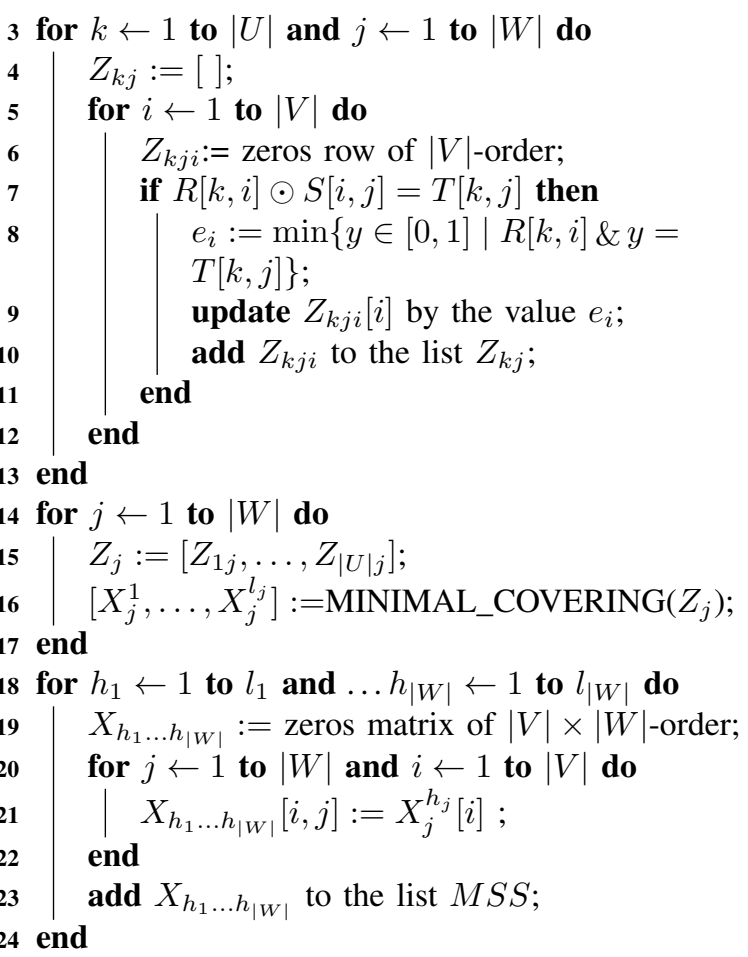

Algorithm 1: $\operatorname{PMINSOLUTIONS}(R, T)$

Given $U=\left\{u_{1}, u_{2}, u_{3}\right\}, V=\left\{v_{1}, v_{2}, v_{3}\right\} W=$ $\left\{w_{1}, w_{2}, w_{3}\right\}$ and the fuzzy relation equations, defined from the following tables

direct computation shows that the relation $R \Rightarrow T$, defined from the table

\begin{tabular}{cccc}
$R \Rightarrow T$ & $w_{1}$ & $w_{2}$ & $w_{3}$ \\
\hline$v_{1}$ & 0.9 & 0.5 & 0.8 \\
$v_{2}$ & 0.7 & 0.8 & 0.4 \\
$v_{3}$ & 0.9 & 0.5 & 0.6
\end{tabular}

is the greatest solution of Equation (2). During the verification we go through the following calculations:

When computing $(R \circ(R \Rightarrow T))\left\langle u_{1}, w_{1}\right\rangle=0.8$, we consider the maximum of

$$
\begin{aligned}
& R\left\langle u_{1}, v_{1}\right\rangle \odot(R \Rightarrow T)\left\langle v_{1}, w_{1}\right\rangle=0.9+0.9-1=0.8 \\
& R\left\langle u_{1}, v_{2}\right\rangle \odot(R \Rightarrow T)\left\langle v_{2}, w_{1}\right\rangle=0.5+0.7-1=0.2 \\
& R\left\langle u_{1}, v_{3}\right\rangle \odot(R \Rightarrow T)\left\langle v_{3}, w_{1}\right\rangle=0.9+0.9-1=0.8
\end{aligned}
$$


Notice that from $v_{1}$ and $v_{3}$ we get the maximum. Hence, in order to obtain this maximum, we only need to consider $\left\{v_{1}\right\}$ or $\left\{v_{3}\right\}$. Moreover, the values 0.9 associated with $v_{1}$ and 0.9 associated with $v_{3}$ cannot be decreased because, if we decrease them, a value less than 0.8 will be obtained in the computation and we do not reach a solution. Therefore, the first column of a solution of Equation (2) could be any column in the set:

$$
Z_{1,1}=\left\{\left(\begin{array}{c}
0.9 \\
0 \\
0
\end{array}\right),\left(\begin{array}{c}
0 \\
0 \\
0.9
\end{array}\right)\right\}
$$

However, we need to verify that the other two equalities also hold. Consequently, the equality $(R \circ(R \Rightarrow T))\left\langle u_{2}, w_{1}\right\rangle=$ 0.6 is studied similarly to the previous procedure. The value $(R \circ(R \Rightarrow T))\left\langle u_{2}, w_{1}\right\rangle$ is the maximum of the values

$$
\begin{aligned}
& R\left\langle u_{2}, v_{1}\right\rangle \odot(R \Rightarrow T)\left\langle v_{1}, w_{1}\right\rangle=0.2+0.9-1=0.1 \\
& R\left\langle u_{2}, v_{2}\right\rangle \odot(R \Rightarrow T)\left\langle v_{2}, w_{1}\right\rangle=0.9+0.7-1=0.6 \\
& R\left\langle u_{2}, v_{3}\right\rangle \odot(R \Rightarrow T)\left\langle v_{3}, w_{1}\right\rangle=0.7+0.9-1=0.6
\end{aligned}
$$

for which $\left\{v_{2}\right\}$ or $\left\{v_{3}\right\}$ is only necessary and so, the first column of a solution of Equation (2) could be one element of the set:

$$
Z_{2,1}=\left\{\left(\begin{array}{c}
0 \\
0.7 \\
0
\end{array}\right),\left(\begin{array}{c}
0 \\
0 \\
0.9
\end{array}\right)\right\}
$$

Finally, when computing $(R \circ(R \Rightarrow T))\left\langle u_{3}, w_{1}\right\rangle=0.8$ we pass by

$$
\begin{aligned}
& R\left\langle u_{3}, v_{1}\right\rangle \odot(R \Rightarrow T)\left\langle v_{1}, w_{1}\right\rangle=0.8+0.9-1=0.7 \\
& R\left\langle u_{3}, v_{2}\right\rangle \odot(R \Rightarrow T)\left\langle v_{2}, w_{1}\right\rangle=0.6+0.7-1=0.3 \\
& R\left\langle u_{3}, v_{3}\right\rangle \odot(R \Rightarrow T)\left\langle v_{3}, w_{1}\right\rangle=0.9+0.9-1=0.8
\end{aligned}
$$

In this case, only $v_{3}$ is necessary and one column is only considered:

$$
Z_{3,1}=\left\{\left(\begin{array}{c}
0 \\
0 \\
0.9
\end{array}\right)\right\}
$$

We observe that

$$
K=\left(\begin{array}{c}
0 \\
0 \\
0.9
\end{array}\right) \in Z_{1,1} \cap Z_{2,1} \cap Z_{3,1},
$$

so $K$ is the only minimal column which, in an intuitive sense, covers the set $Z_{1}=\left\{Z_{1,1}, Z_{2,1}, Z_{3,1}\right\}$. Moreover, we conclude that a fuzzy relation $X_{1}$, defined as

\begin{tabular}{cccc}
$X_{1}$ & $w_{1}$ & $w_{2}$ & $w_{3}$ \\
\hline$v_{1}$ & 0 & 0.5 & 0.8 \\
$v_{2}$ & 0 & 0.8 & 0.4 \\
$v_{3}$ & 0.9 & 0.5 & 0.6
\end{tabular}

solves the fuzzy relation equation (2).

Next, we consider the second column of $R \Rightarrow T$, which provides a different case. For $(R \circ(R \Rightarrow T))\left\langle u_{1}, w_{2}\right\rangle=0.4$ we have

$$
\begin{aligned}
& R\left\langle u_{1}, v_{1}\right\rangle \odot(R \Rightarrow T)\left\langle v_{1}, w_{2}\right\rangle=0.9+0.5-1=0.4 \\
& R\left\langle u_{1}, v_{2}\right\rangle \odot(R \Rightarrow T)\left\langle v_{2}, w_{2}\right\rangle=0.5+0.8-1=0.3 \\
& R\left\langle u_{1}, v_{3}\right\rangle \odot(R \Rightarrow T)\left\langle v_{3}, w_{2}\right\rangle=0.9+0.5-1=0.4
\end{aligned}
$$

Hence, the maximum is obtained from $v_{1}$ or $v_{3}$ and, therefore, the following set is considered:

$$
Z_{1,2}=\left\{\left(\begin{array}{c}
0.5 \\
0 \\
0
\end{array}\right),\left(\begin{array}{c}
0 \\
0 \\
0.5
\end{array}\right)\right\}
$$

For $(R \circ(R \Rightarrow T))\left\langle u_{2}, w_{2}\right\rangle=0.7$ we have

$$
\begin{aligned}
& R\left\langle u_{2}, v_{1}\right\rangle \odot(R \Rightarrow T)\left\langle v_{1}, w_{2}\right\rangle=0 \\
& R\left\langle u_{2}, v_{2}\right\rangle \odot(R \Rightarrow T)\left\langle v_{2}, w_{2}\right\rangle=0.9+0.8-1=0.7 \\
& R\left\langle u_{2}, v_{3}\right\rangle \odot(R \Rightarrow T)\left\langle v_{3}, w_{2}\right\rangle=0.7+0.5-1=0.2
\end{aligned}
$$

Consequently, the subset obtained is

$$
Z_{2,2}=\left\{\left(\begin{array}{c}
0 \\
0.8 \\
0
\end{array}\right)\right\}
$$

For $(R \circ(R \Rightarrow T))\left\langle u_{3}, w_{2}\right\rangle=0.4$ we have

$$
\begin{aligned}
& R\left\langle u_{3}, v_{1}\right\rangle \odot(R \Rightarrow T)\left\langle v_{1}, w_{2}\right\rangle=0.8+0.5-1=0.3 \\
& R\left\langle u_{3}, v_{2}\right\rangle \odot(R \Rightarrow T)\left\langle v_{2}, w_{2}\right\rangle=0.6+0.8-1=0.4 \\
& R\left\langle u_{3}, v_{3}\right\rangle \odot(R \Rightarrow T)\left\langle v_{3}, w_{2}\right\rangle=0.9+0.5-1=0.4
\end{aligned}
$$

Hence, the assumed subset of columns is

$$
Z_{3,2}=\left\{\left(\begin{array}{c}
0 \\
0 \\
0.5
\end{array}\right),\left(\begin{array}{c}
0 \\
0.8 \\
0
\end{array}\right)\right\}
$$

In this case, we observe that $Z_{1,2} \cap Z_{2,2} \cap Z_{3,2}=\varnothing$. However,

$$
\begin{aligned}
& \left(\begin{array}{c}
0 \\
0 \\
0.5
\end{array}\right),\left(\begin{array}{c}
0 \\
0.8 \\
0
\end{array}\right) \leq\left(\begin{array}{c}
0 \\
0.8 \\
0.5
\end{array}\right)=\left(\begin{array}{c}
0 \\
0.8 \\
0
\end{array}\right) \vee\left(\begin{array}{c}
0 \\
0 \\
0.5
\end{array}\right), \\
& \left(\begin{array}{c}
0.5 \\
0 \\
0 .
\end{array}\right),\left(\begin{array}{c}
0 \\
0.8 \\
0
\end{array}\right) \leq\left(\begin{array}{c}
0.5 \\
0.8 \\
0
\end{array}\right)=\left(\begin{array}{c}
0 \\
0.8 \\
0
\end{array}\right) \vee\left(\begin{array}{c}
0.5 \\
0 \\
0
\end{array}\right)
\end{aligned}
$$

and $\left(\begin{array}{c}0 \\ 0.8 \\ 0.5\end{array}\right),\left(\begin{array}{c}0.5 \\ 0.8 \\ 0\end{array}\right)$ are the only minimal columns which, again in an intuitive sense, cover the set $Z_{2}=$ $\left\{Z_{1,2}, Z_{2,2}, Z_{3,2}\right\}$. Moreover, we conclude that the fuzzy relations $X_{2}$ and $X_{3}$, defined as

\begin{tabular}{cccc}
$X_{2}$ & $w_{1}$ & $w_{2}$ & $w_{3}$ \\
\hline$v_{1}$ & 0 & 0 & 0.8 \\
$v_{2}$ & 0 & 0.8 & 0.4 \\
$v_{3}$ & 0.9 & 0.5 & 0.6
\end{tabular}

\begin{tabular}{cccc}
$X_{3}$ & $w_{1}$ & $w_{2}$ & $w_{3}$ \\
\hline$v_{1}$ & 0 & 0.5 & 0.8 \\
$v_{2}$ & 0 & 0.8 & 0.4 \\
$v_{3}$ & 0.9 & 0 & 0.6
\end{tabular}

solve the fuzzy relation (2). Finally, the values in the third column of $R \Rightarrow T$ are reduced.

For $(R \circ(R \Rightarrow T))\left\langle u_{1}, w_{3}\right\rangle=0.7$, we compute

$R\left\langle u_{1}, v_{1}\right\rangle \odot(R \Rightarrow T)\left\langle v_{1}, w_{3}\right\rangle=0.9+0.8-1=0.7$

$R\left\langle u_{1}, v_{2}\right\rangle \odot(R \Rightarrow T)\left\langle v_{2}, w_{3}\right\rangle=0$

$R\left\langle u_{1}, v_{3}\right\rangle \odot(R \Rightarrow T)\left\langle v_{3}, w_{3}\right\rangle=0.9+0.6-1=0.5$ 
Hence, $Z_{1,3}=\left\{\left(\begin{array}{c}0.8 \\ 0 \\ 0\end{array}\right)\right\}$.

For $(R \circ(R \Rightarrow T))\left\langle u_{2}, w_{3}\right\rangle=0.3$, we have

$$
\begin{aligned}
& R\left\langle u_{2}, v_{1}\right\rangle \odot(R \Rightarrow T)\left\langle v_{1}, w_{3}\right\rangle=0.2+0.8-1=0 \\
& R\left\langle u_{2}, v_{2}\right\rangle \odot(R \Rightarrow T)\left\langle v_{2}, w_{3}\right\rangle=0.9+0.4-1=0.3 \\
& R\left\langle u_{2}, v_{3}\right\rangle \odot(R \Rightarrow T)\left\langle v_{3}, w_{3}\right\rangle=0.7+0.6-1=0.3
\end{aligned}
$$

two possibilities providing two columns: $Z_{2,3}=$ $\left\{\left(\begin{array}{c}0 \\ 0.4 \\ 0\end{array}\right),\left(\begin{array}{c}0 \\ 0 \\ 0.6\end{array}\right)\right\}$

For $(R \circ(R \Rightarrow T))\left\langle u_{3}, w_{3}\right\rangle=0.6$ we have

$R\left\langle u_{3}, v_{1}\right\rangle \odot(R \Rightarrow T)\left\langle v_{1}, w_{3}\right\rangle=0.8+0.8-1=0.6$

$R\left\langle u_{3}, v_{2}\right\rangle \odot(R \Rightarrow T)\left\langle v_{2}, w_{3}\right\rangle=0.6+0.4-1=0$

$R\left\langle u_{3}, v_{3}\right\rangle \odot(R \Rightarrow T)\left\langle v_{3}, w_{3}\right\rangle=0.9+0.6-1=0.5$

Therefore, $Z_{3,3}=\left\{\left(\begin{array}{c}0.8 \\ 0 \\ 0\end{array}\right)\right\}$.

In this case, there are two minimal covering of the set $Z_{3}=$ $\left\{Z_{1,3}, Z_{2,3}, Z_{3,3}\right\}$ :

$\left(\begin{array}{c}0.8 \\ 0.4 \\ 0\end{array}\right)=\left(\begin{array}{c}0.8 \\ 0 \\ 0\end{array}\right) \vee\left(\begin{array}{c}0 \\ 0.4 \\ 0\end{array}\right)$ and $\left(\begin{array}{c}0.8 \\ 0 \\ 0.6\end{array}\right)=\left(\begin{array}{c}0.8 \\ 0 \\ 0\end{array}\right) \vee\left(\begin{array}{c}0 \\ 0 \\ 0.6\end{array}\right)$

This yields four fuzzy relations, defined as follows

\begin{tabular}{cccc}
$X_{4}$ & $w_{1}$ & $w_{2}$ & $w_{3}$ \\
\hline$v_{1}$ & 0 & 0 & 0.8 \\
$v_{2}$ & 0 & 0.8 & 0.4 \\
$v_{3}$ & 0.9 & 0.5 & 0 \\
$X_{6}$ & $w_{1}$ & $w_{2}$ & $w_{3}$ \\
\hline$v_{1}$ & 0 & 0.5 & 0.8 \\
$v_{2}$ & 0 & 0.8 & 0.4 \\
$v_{3}$ & 0.9 & 0 & 0
\end{tabular}

\begin{tabular}{cccc}
$X_{5}$ & $w_{1}$ & $w_{2}$ & $w_{3}$ \\
\hline$v_{1}$ & 0 & 0 & 0.8 \\
$v_{2}$ & 0 & 0.8 & 0 \\
$v_{3}$ & 0.9 & 0.5 & 0.6 \\
$X_{7}$ & $w_{1}$ & $w_{2}$ & $w_{3}$ \\
\hline$v_{1}$ & 0 & 0.5 & 0.8 \\
$v_{2}$ & 0 & 0.8 & 0 \\
$v_{3}$ & 0.9 & 0 & 0.6
\end{tabular}

that solve Equation (2). By their construction and the properties of the Łukasiewicz conjunctor, they are minimal solutions.

Example III.2. In this example, we consider the Gödel structure [9], then $L=[0,1]$ and $\odot: L \times L \rightarrow L$ and $\rightarrow: L \times L \rightarrow L$ are defined by $x \odot y=\min \{x, y\}$ and

$$
y \rightarrow z= \begin{cases}1 & \text { if } y \leq z \\ z & \text { otherwise }\end{cases}
$$

for all $x, y, z \in[0,1]$. Given $U=\left\{u_{1}, u_{2}\right\}, V=\left\{v_{1}, v_{2}, v_{3}\right\}$, $W=\{w\}$ and

\begin{tabular}{ccccccc}
$R$ & $v_{1}$ & $v_{2}$ & $v_{3}$ & & $T$ & $w$ \\
\hline$u_{1}$ & 0.6 & 0.4 & 0.5 & & $u_{1}$ & 0.6 \\
$u_{2}$ & 0.8 & 0.7 & 0.6 & & $u_{2}$ & 0.7 \\
$u_{3}$ & 0.9 & 1 & 0.9 & & $u_{3}$ & 0.9
\end{tabular}

the direct computation shows that

\begin{tabular}{cc}
$R \Rightarrow T$ & $w$ \\
\hline$v_{1}$ & 0.7 \\
$v_{2}$ & 0.9 \\
$v_{3}$ & 1.0
\end{tabular}

is the maximal solution of Equation (2). In order to verify the equality $(R \circ(R \Rightarrow T))\left\langle u_{1}, w\right\rangle=0.6$ we compute

$$
\begin{aligned}
& R\left\langle u_{1}, v_{1}\right\rangle \odot(R \Rightarrow T)\left\langle v_{1}, w\right\rangle=0.6 \wedge 0.7=0.6 \\
& R\left\langle u_{1}, v_{2}\right\rangle \odot(R \Rightarrow T)\left\langle v_{2}, w\right\rangle=0.4 \wedge 0.9=0.4 \\
& R\left\langle u_{1}, v_{3}\right\rangle \odot(R \Rightarrow T)\left\langle v_{2}, w\right\rangle=0.5 \wedge 1.0=0.5
\end{aligned}
$$

Note that we only need the value associated with $v_{1}$. Moreover, this value can be reduced until 0.6. Hence, the first (and only) column of a solution has to be contained in the following set

$$
Z_{1,1}=\left\{\left(\begin{array}{l}
x \\
0 \\
0
\end{array}\right) \mid 0.6 \leq x \leq 1\right\}
$$

Focusing on our main goal, the least one is the column associated with a minimal solution. Hence, we only consider the column $Z_{1,1}=\left\{\left(\begin{array}{c}0.6 \\ 0 \\ 0\end{array}\right)\right\}$

For $(R \circ(R \Rightarrow T))\left\langle u_{2}, w\right\rangle=0.7$ we have

$$
\begin{aligned}
& R\left\langle u_{2}, v_{1}\right\rangle \odot(R \Rightarrow T)\left\langle v_{1}, w\right\rangle=0.8 \wedge 0.7=0.7 \\
& R\left\langle u_{2}, v_{2}\right\rangle \odot(R \Rightarrow T)\left\langle v_{2}, w\right\rangle=0.7 \wedge 0.9=0.7 \\
& R\left\langle u_{2}, v_{3}\right\rangle \odot(R \Rightarrow T)\left\langle v_{3}, w\right\rangle=0.6 \wedge 1.0=0.6
\end{aligned}
$$

Now the values associated with $v_{1}$ and $v_{2}$ provide the maximum. Furthermore, the value for $v_{2}$ can also be decreased, specifically, any element $x$ in $[0.7,1]$ provides the same maximum result: $0.7 \wedge x=0.7$. Therefore, focusing on the minimal solutions we only need to consider:

$$
Z_{2,1}=\left\{\left(\begin{array}{c}
0.7 \\
0 \\
0
\end{array}\right),\left(\begin{array}{c}
0 \\
0.7 \\
0
\end{array}\right)\right\}
$$

Finally, for $(R \circ(R \Rightarrow T))\left\langle u_{3}, w\right\rangle=0.9$ we calculate

$$
\begin{aligned}
& R\left\langle u_{3}, v_{1}\right\rangle \odot(R \Rightarrow T)\left\langle v_{1}, w\right\rangle=0.9 \wedge 0.7=0.7 \\
& R\left\langle u_{3}, v_{2}\right\rangle \odot(R \Rightarrow T)\left\langle v_{2}, w\right\rangle=1.0 \wedge 0.9=0.9 \\
& R\left\langle u_{3}, v_{3}\right\rangle \odot(R \Rightarrow T)\left\langle v_{3}, w\right\rangle=0.9 \wedge 1.0=0.9
\end{aligned}
$$

In this last case $v_{2}$ and $v_{3}$ are involved in the computation of the maximum and the value associated with $v_{3}$ can be decreased until 0.9. These considerations yield the following minimal solutions

$$
\left(\begin{array}{l}
0.6 \\
0.7 \\
0.9
\end{array}\right),\left(\begin{array}{c}
0.7 \\
0 \\
0.9
\end{array}\right),\left(\begin{array}{c}
0.6 \\
0.9 \\
0
\end{array}\right)
$$

\section{CONCLUSION AND FUTURE WORKS}

The main aim of this research is to define as generally as possible an algebraic structure that allows the existence of minimal solutions of the fuzzy relation equations defined based on this structure. For that, a general increasing operation $\odot$, which only satisfies the adjointness property, i.e. is residuated, and satisfies the IPNE-condition, has been considered to define a general fuzzy relation equation, which has minimal solutions whenever a solution exists. Moreover, a new algebraic characterization using the notion of covering is introduced, 
which provides a method to obtain the minimal solutions and, consequently, the whole set of solutions.

As future work, the obtained results will be applied to several problems in fuzzy logic, such as to abduction reasoning. It is well-known that implications in MV-algebras are infinitely distributive. A topic of future study is to characterize all structures where implication is infinitely distributivity. Algebraic structures that satisfy the INPE-condition are not studied much; also they will be a topic of future research.

\section{REFERENCES}

[1] W. Bandler and L. Kohout. Semantics of implication operators and fuzzy relational products. Int. J. Man-Machine Studies, 12:89-116, 1980.

[2] E. Bartl. Minimal solutions of generalized fuzzy relational equations: Probabilistic algorithm based on greedy approach. Fuzzy Sets and Systems, 260(0):25 - 42, 2015.

[3] R. Bělohlávek. Fuzzy Relational Systems: Foundations and Principles. Kluwer Academic Publishers, 2002.

[4] L. Chen and P. Wang. Fuzzy relation equations (ii): The branchpoint-solutions and the categorized minimal solutions. Soft ComputingA Fusion of Foundations, Methodologies and Applications, 11:33-40, 2007.

[5] M. E. Cornejo, J. Medina, and E. Ramírez-Poussa. Multi-adjoint algebras versus non-commutative residuated structures. International Journal of Approximate Reasoning, 66:119-138, 2015.

[6] B. De Baets. Analytical solution methods for fuzzy relation equations In D. Dubois and H. Prade, editors, The Handbooks of Fuzzy Sets Series, volume 1, pages 291-340. Kluwer, Dordrecht, 1999.
[7] J. C. Díaz-Moreno, J. Medina, and M. Ojeda-Aciego. On basic conditions to generate multi-adjoint concept lattices via galois connections. International Journal of General Systems, 43(2):149-161, 2014.

[8] J. C. Díaz-Moreno, J. Medina, and E. Turunen. Minimal solutions of general fuzzy relation equations on linear carriers. an algebraic characterization. Fuzzy Sets and Systems, pages -, 2016.

[9] P. Hájek. Metamathematics of Fuzzy Logic. Trends in Logic. Kluwer Academic, 1998.

[10] J.-L. Lin, Y.-K. Wu, and S.-M. Guu. On fuzzy relational equations and the covering problem. Information Sciences, 181(14):2951-2963, 2011.

[11] E. Sanchez. Resolution of composite fuzzy relation equations. Information and Control, 30(1):38-48, 1976.

[12] B.-S. Shieh. Solution to the covering problem. Information Sciences, 222(0):626-633, 2013.

[13] E. Shivanian. An algorithm for finding solutions of fuzzy relation equations with max-lukasiewicz composition. Mathware and Soft Computing, 17:15-26, 2010.

[14] E. Turunen. On generalized fuzzy relation equations: necessary and sufficient conditions for the existence of solutions. Acta Universitatis Carolinae. Mathematica et Physica, 028(1):33-37, 1987.

[15] Q.-Q. Xiong and X.-P. Wang. Solution sets of fuzzy relational equations on complete Brouwerian lattices. Information Sciences, 177(21):47574767, 2007.

[16] Q.-Q. Xiong and X.-P. Wang. Fuzzy relational equations on complete Brouwerian lattices. Information Sciences, 193(0):141-152, 2012.

[17] C.-T. Yeh. On the minimal solutions of max-min fuzzy relational equations. Fuzzy Sets and Systems, 159(1):23-39, 2008.

[18] Z. Zahariev. http://www.mathworks.com/matlabcentral, fuzzy-calculus-core-fc2ore, 2010. 